\title{
Rotor Position and Speed Estimation Method for Magnetically Suspended PMSM Based on Modified Sliding Mode Observer
}

\author{
Haicai Liu and Guangjun $\mathrm{Li}^{*}$ \\ Beihang University, School of Mechanical Engineering and Automation, China \\ ${ }^{*}$ Corresponding author
}

\begin{abstract}
This paper proposes a method of rotor position and speed estimation for magnetically suspended permanent magnetic synchronous motor (PMSM) based on modified sliding mode observer(SMO). In order to weaken the chatting phenomenon, the estimated back-EMF is brought in the current model and a hyperbolic tangent function is used. In order eliminate the high-order harmonic components in the estimated back-EMF, an adaptive low-pass filter whose cutoff frequency varies with the motor electrical frequency is adopted. The stability of the algorithm is proved by the Lyapunov stability theory. Finally, the validity of the method is verified by simulation and experiment.
\end{abstract}

Keywords-magnetic flywheel energy storage system; permanent magnetic synchronous motor; sliding mode observer; lyapunov; adaptive low-pass filter

\section{INTRODUCTION}

Magnetically suspended flywheel(MSFW) energy storage system consists of flywheel rotor, magnetic bearing, motor and power electronic equipment which transforms electric energy to kinetic energy[1-2]. Because PMSM have the advantages of high power density, high efficiency and little consumption, it is widely applied in the field of flywheel energy storage system(FESS)[3-8]. Usually the rotor's position of the PMSM is measured by an optical encoder or a resolver, but it increases the system cost and bulk, and reduces the reliability. Therefore accurate sensorless position and speed estimation method is of great significance to solve the above problems.

The vector control based on sliding mode observer has been widely used in PMSM sensorless control, and has been concerned by scholars. At present, the main research work includes sliding mode observer, low-pass filter, and speed identification. The paper [9] proposed a iterative sliding mode observer with a sigmoid to reduce the chatting phenomenon, however the estimated back-EMF is always corrupted by harmonics because of the gap between PMs and inverter nonlinearity, the flux spatial harmonics, but it didn't have a filter. The paper [10] introduced a high order sliding mode controller based on complete model of the surface PMSM, but the algorithms require large computations and result in cycle time longer for FESS. The paper [11] and [12] applied phaselocked loop to decrease the chatting problem, and it was proved well.
Based on the analysis of existing research, a kind of magnetically suspended PMSM rotor position and speed estimation method for the magnetic flywheel energy storage system based on a modified sliding mode observer is proposed. The estimated back EMF is used in the current model of the modified SMO. And a hyperbolic tangent function and an adaptive low-pass filter are used to reduce the chatting. Finally, the validity of the method is verified by simulation and experiment.

\section{DYNAMIC MODEL OF A PMSM}

Based on the following assumptions: sinusoidal symmetrical distribution of stator winding magnetomotive force; saturation and parameter changes are neglected, the current model of the nonsalient surface PMSM in the twopahse stationary reference frame is described by[13]

$$
\begin{aligned}
& \left\{\begin{array}{l}
\frac{d i_{\alpha}}{d t}=-\frac{R_{s}}{L_{s}} i_{\alpha}-\frac{1}{L_{s}} e_{\alpha}+\frac{1}{L_{s}} u_{\alpha} \\
\frac{d i_{\beta}}{d t}=-\frac{R_{s}}{L_{s}} i_{\beta}-\frac{1}{L_{s}} e_{\beta}+\frac{1}{L_{s}} u_{\beta}
\end{array}\right. \\
& \left\{\begin{array}{l}
e_{\alpha}=-\psi_{\mathrm{f}} \omega_{\mathrm{r}} \sin \theta \\
e_{\beta}=\psi_{\mathrm{f}} \omega_{\mathrm{r}} \sin \theta
\end{array}\right.
\end{aligned}
$$

where $i_{\alpha}$ and $i_{\beta}$ are the stator current, $e_{\alpha}$ and $e_{\beta}$ are the back EMF, $u_{\alpha}$ and $u_{\beta}$ are the stator voltage, $R_{s}$ and $L_{s}$ represent the stator resistance and inductance. $\psi_{\mathrm{f}}$ is the amplitudes of permanent magnetic flux linkage, $\omega_{\mathrm{r}}$ is the electrical angular velocity, respectively.

\section{DESIGN OF THE MODIEIED SMO FOR PMSM}

The conventional SMO which uses a constant switch function is described by[14][15] 


$$
\left\{\begin{array}{l}
\frac{d \hat{i}_{\alpha}}{d t}=-\frac{R_{s}}{L_{s}} \hat{i}_{\alpha}+\frac{1}{L_{s}} u_{\alpha}-z_{t \alpha} \\
\frac{d \hat{i}_{\beta}}{d t}=-\frac{R_{s}}{L_{s}} \hat{i}_{\beta}+\frac{1}{L_{s}} u_{\beta}-z_{t \beta}
\end{array}\right.
$$

where $\hat{i}_{\alpha}$ and $\hat{i}_{\beta}$ are stator current estimation value, $z_{\text {t } \alpha}$ and $Z_{t \beta}$ are the switch function of conventional SMO.

$$
\left\{\begin{array}{l}
z_{t \alpha}=k_{t} \cdot \operatorname{sgn}\left(\hat{i}_{\alpha}-i_{\alpha}\right) \\
z_{t \beta}=k_{t} \cdot \operatorname{sgn}\left(\hat{i}_{\beta}-i_{\beta}\right)
\end{array}\right.
$$

where $k_{t}>0, k_{t}$ is the sliding-mode gain. Figure I displays the block diagram of the traditional SMO.

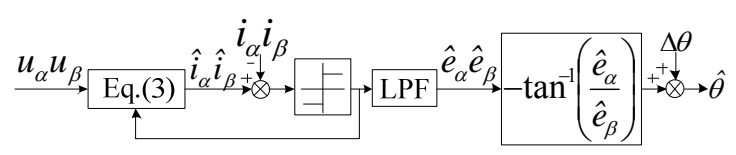

FIGURE I. CONVENTIONAL SLIDING MODE OBSERVER

The ideal switching characteristics of (4) is difficult to be obtained in the MSFW energy storage system. Due to the time delay and spatial lag, sliding mode motion has the chatting problem. In order to decrease the chatting, the current model of the modified SMO is expressed as

$$
\left\{\begin{array}{l}
\frac{d \hat{i}_{\alpha}}{d t}=-\frac{R_{s}}{L_{s}} \hat{i}_{\alpha}-\frac{1}{L_{s}} \hat{e}_{\alpha}+\frac{1}{L_{s}} u_{\alpha}-z_{\alpha} \\
\frac{d \hat{i}_{\beta}}{d t}=-\frac{R_{s}}{L_{s}} \hat{i}_{\beta}-\frac{1}{L_{s}} \hat{e}_{\beta}+\frac{1}{L_{s}} u_{\beta}-z_{\beta}
\end{array}\right.
$$

where $Z_{\alpha}$ and $Z_{\beta}$ are the switch function.

$$
\left\{\begin{array}{l}
\mathrm{z}_{\alpha}=k \cdot \tanh \left(\frac{s_{\alpha}}{\varepsilon}\right) \\
\mathrm{z}_{\beta}=k \cdot \tanh \left(\frac{s_{\beta}}{\varepsilon}\right)
\end{array}\right.
$$

where $k>0, k$ is the sliding mode gain of the modified $\mathrm{SMO}, \varepsilon$ is the parameter of the modified SMO.

The sliding surface is defined as

$$
S=\left[\begin{array}{l}
s_{\alpha} \\
s_{\beta}
\end{array}\right]=\left[\begin{array}{l}
\hat{i}_{\alpha}-i_{\alpha} \\
\hat{i}_{\beta}-i_{\beta}
\end{array}\right]
$$

Figure II shows the curve of switch function. When $\varepsilon$ is appropriately increased, the derivative of the origin is reduced.
Then the sliding mode speed is smaller near the sliding surface which can decrease the chatting and delay the system dynamic response.

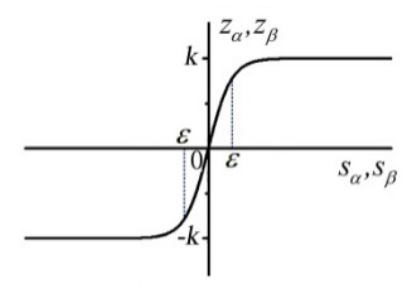

FIGURE II. CURVE OF SWITCH FUNCTION

The back EMF is estimated by the adaptive low-pass filter from the switch function, shown as follow

$$
\left\{\begin{array}{l}
\frac{d \hat{e}_{\alpha}}{d t}=-2 \pi \omega_{c} \hat{e}_{\alpha}+2 \pi \omega_{c} z_{\alpha} \\
\frac{d \hat{e}_{\beta}}{d t}=-2 \pi \omega_{c} \hat{e}_{\beta}+2 \pi \omega_{c} z_{\beta}
\end{array}\right.
$$

where $\omega_{c}$ represents the cutoff frequency.

In order to improve the low-pass filter, $\omega_{c}$ varies with $\omega_{e}\left(\omega_{e}\right.$ represents electrical angle frequency), which can be described by

$$
\omega_{c}=l \omega_{e}
$$

where $l$ is the parameter of the filter.

From (2), we obtain the estimated rotor position which is described by

$$
\theta=-\arctan \left(\frac{e_{\alpha}}{e_{\beta}}\right)
$$

The compensation angle is used for the delay of the rotor position caused by the low-pass filter, expressed as

$$
\Delta \theta=\arctan \left(\frac{\hat{\omega}}{\omega_{c}}\right)
$$

From (8) and (10), the estimated rotor position with the compensation angle can be expressed as follow:

$$
\hat{\theta}=\theta+\Delta \theta=-\arctan \left(\frac{e_{\alpha}}{e_{\beta}}\right)+\arctan \left(\frac{\hat{\omega}}{\omega_{0}}\right)
$$

Rotor speed can be described by 


$$
\hat{\omega}=\frac{d \hat{\theta}}{d t}
$$

In order to reduce the amplifying noise generated by the pure differentiator, the rotor speed is necessary to be filtered out by the low-pass filter. By comprehensive consideration, the block diagram of the modified SMO is displayed in Figure III.

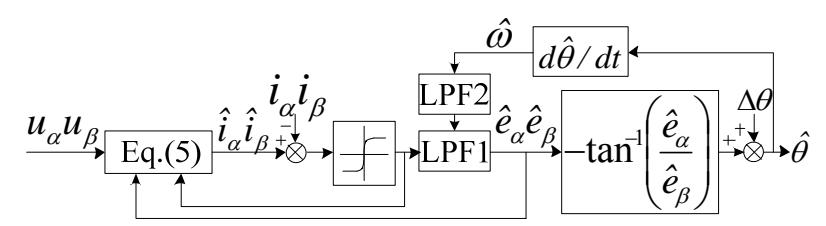

FIGURE III. BLOCK DIAGRAM OF THE MODIFIED SMO

Figure III shows the block diagram of the modified SMO whose stability and convergence are demonstrated in the next section.

\section{STABILITY ANALYSIS}

The current estimation errors is 0 at the time of reaching the sliding surface where the SMO shows good robustness against the system parameters.

In order to design a stable observer, the Lyapunov function used to find the sliding-mode condition can be defined as

$$
V=\frac{1}{2} S^{\mathrm{T}} S
$$

From the Lyapunov stability theorem, the sliding mode condition can be derived to satisfy the conditions $V>0$ and $\dot{V}<0$. (5) substract (1), we can obtain

$$
\left\{\begin{array}{l}
\frac{d s_{\alpha}}{d t}=-\frac{R_{s}}{L_{s}} s_{\alpha}-\frac{1}{L_{s}}\left(\hat{e}_{\alpha}-e_{\alpha}\right)-k \cdot \tanh \left(\frac{s_{\alpha}}{\varepsilon}\right) \\
\frac{d s_{\beta}}{d t}=-\frac{R_{s}}{L_{s}} s_{\beta}-\frac{1}{L_{s}}\left(\hat{e}_{\beta}-e_{\beta}\right)-k \cdot \tanh \left(\frac{s_{\beta}}{\varepsilon}\right)
\end{array}\right.
$$

From (7) and (14), we can obtain

$V=\frac{1}{2}\left[\left(\hat{i}_{\alpha}-i_{\alpha}\right)^{2}+\left(\hat{i}_{\beta}-i_{\beta}\right)^{2}\right] \geq 0$, where equal only on the sliding surface.

From (7), (14) and (15), we can get (16) and (17).

$$
\dot{V}=S^{\mathrm{T}} \dot{S}=\left[\begin{array}{ll}
S_{\alpha} & s_{\beta}
\end{array}\right] \cdot\left[\begin{array}{c}
\dot{S}_{\alpha} \\
\dot{S}_{\beta}
\end{array}\right]=s_{\alpha} \dot{S}_{\alpha}+s_{\beta} \dot{S}_{\beta}
$$

In (16)

$$
\left\{\begin{array}{l}
s_{\alpha} \dot{s}_{\alpha}=-\frac{R_{s}}{L_{s}} s_{\alpha}{ }^{2}-\frac{1}{L_{s}}\left(\hat{e}_{\alpha}-e_{\alpha}\right)-k \cdot \tanh \left(\frac{s_{\alpha}}{\varepsilon}\right) \cdot s_{\alpha} \\
s_{\beta} \dot{s}_{\beta}=-\frac{R_{s}}{L_{s}} s_{\beta}{ }^{2}-\frac{1}{L_{s}}\left(\hat{e}_{\beta}-e_{\beta}\right)-k \cdot \tanh \left(\frac{s_{\beta}}{\varepsilon}\right) \cdot s_{\beta}
\end{array}\right.
$$

where $-\frac{R_{s}}{L_{s}} S_{\alpha}^{2}<0,-\frac{R_{s}}{L_{s}} S_{\beta}^{2}<0$. If

$$
k=\left(\frac{\left|\hat{e}_{\alpha}-e_{\alpha}\right|}{L_{s} \cdot s_{\alpha} \cdot \tanh \left(\frac{s_{\alpha}}{\varepsilon}\right)}, \frac{\left|\hat{e}_{\beta}-e_{\beta}\right|}{L_{s} \cdot s_{\beta} \cdot \tanh \left(\frac{s_{\beta}}{\varepsilon}\right)}\right) \max , s_{\alpha} \dot{s}_{\alpha}<0
$$

and $s_{\beta} \dot{s}_{\beta}<0$. So $\dot{V}<0$, and the system is proved to be stable.

\section{Simulation AND EXPERIMENTAL RESUltS}

\section{A. Simulation results}

In order to verify the validity of the proposed SMO, a Matlab/Simulink simulation model is developed as shown in Figure IV. The parameters of simulation motor shown in Table 1 is same to the parameters of magnetic levitation PMSM and the reference speed is $600 \mathrm{r} / \mathrm{min}$.

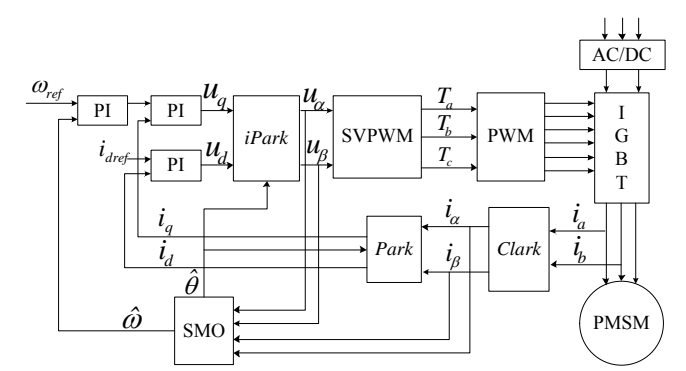

FIGURE IV. BLOCK DIAGRAM OF THE VECTOR CONTROL

TABLE I. PARAMETERS OF MAGNETIC LEVITATION PMSM

\begin{tabular}{ll}
\hline Rated power & $300 \mathrm{~kW}$ \\
Rated speed & $30000 \mathrm{r} / \mathrm{min}$ \\
BEMF coefficient & $0.5 \mathrm{~V} /(\mathrm{kr} / \mathrm{min})$ \\
Flux linkage & $0.03 \mathrm{~Wb}$ \\
Pole pairs number & 1 \\
Stator resistance & $0.14 \Omega$ \\
Stator inductance & $0.3 \mathrm{mH}$ \\
Moment of inertia & $1.47 \mathrm{~kg} \cdot \mathrm{m}^{2}$ \\
\hline
\end{tabular}

Figure $\mathrm{V}(\mathrm{a})$ shows that the rotor positon estimated by traditional SMO has the obvious chatting phenomenon whlie induced chatting estimated by modified SMO. From Figure $\mathrm{V}(\mathrm{b})$, we can know that the speed curve with a big wave was estimed by traditional SMO. However it is smooth near the real speed required by modified SMO. 


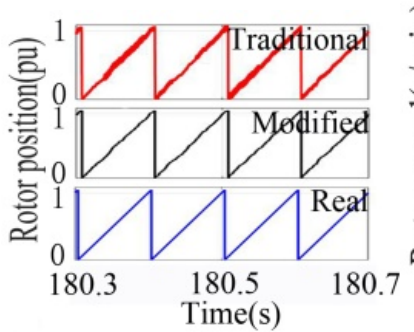

(a)

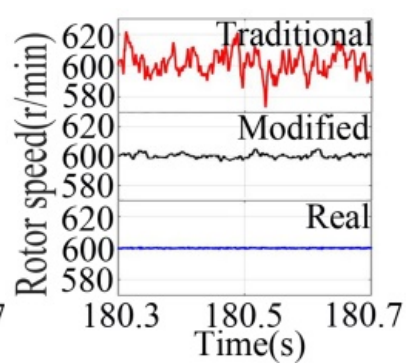

(b)
FIGURE V. SIMULATION RESULTS AT THE SPEED OF 600R/MIN(A) ROTOR POSITION(B) ROTOR SPEED

\section{EXPERIMENTAL RESULTS}

Figure VI displays the MSFW energy storage system, where some experiments were carried on to verfy the validity of the modified SMO. The model of DSP is TMS320F23885 of TI company and the PWM frequency is $10 \mathrm{kHz}$. The DSP transfer information to host computer by CAN bus with $1 \mathrm{kHz}$ rate. The parameters of PMSM is shown in Table 1, and the reference speeds are $600 \mathrm{r} / \mathrm{min}$ and $3000 \mathrm{r} / \mathrm{min}$. Experimental resullts are displayed in Figure VII and Figure VIII with the $i_{d}=0$ vector control.

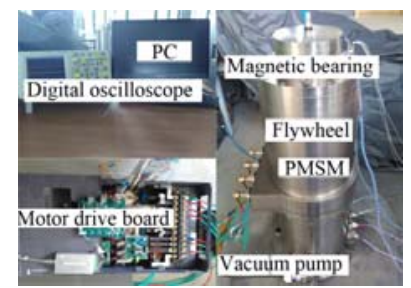

FIGURE VI. THE MAGNETIC FLYWHEEL ENERGY STORAGE SYSTEM

From Figure VII, the rotor position estimated by modified SMO is extremely near to real rotor position with a little wave at the speed of $600 \mathrm{r} / \mathrm{min}$ and $3000 \mathrm{r} / \mathrm{min}$.

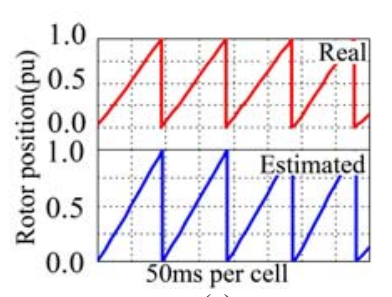

(a)

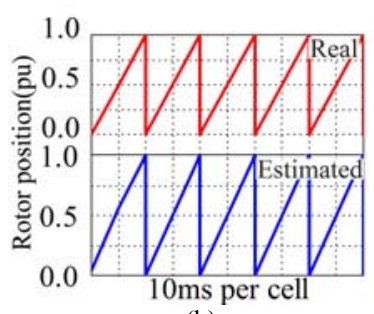

(b)
FIGURE VII. ROTOR POSITION (A) 600R/MIN (B) 3000R/MIN

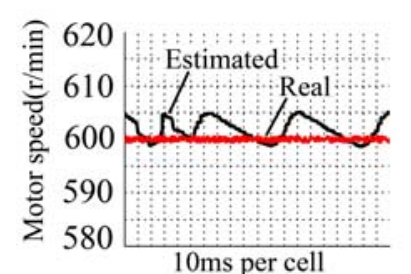

(a)

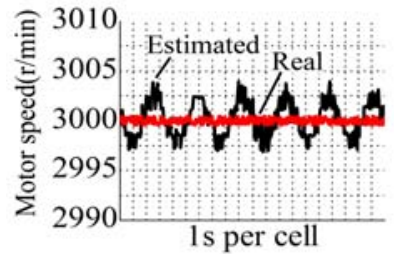

(b)
FIGURE VIII. MOTOR SPEED (A) 600R/MIN(B) 3000R/MIN
From Figure VIII(a), there are small fluctuations in the curve of PMSM rotor speed $600 \mathrm{r} / \mathrm{min}$ required by the modified SMO. The average of the speed is higher than reference speed $2.5 \mathrm{r} / \mathrm{min}$ and the range of the speed error is $2 \sim+5 \mathrm{r} / \mathrm{min}$ which falls within acceptable limits. From Figure VIII(b), we can know that the speed curve estimated by the modified SMO has a extremely small wave. The avarage of estimated speed is same to the real speed and the range of estimated speed error is $-3 \sim+4 \mathrm{r} / \mathrm{min}$ which is acceptable. Comparing Figure VIII(a) and VIII(b), we can find out that it has a better performance on the avarage of estimated speed and range of speed error at the speed of $3000 \mathrm{r} / \mathrm{min}$. The higher motor speed, the higher back EMF. The estimated error of back EMF is smaller so the rotor position and motor speed calculated from the back EMF is more accurate.

\section{CONLUSION}

This paper has proposed a novel rotor position and speed measuring method based on the modified SMO. Estimated back EMF and a hyperbolic tangent function are used to induced the chatting. In order to filter out harmonics, an adaptive low-pass filter is utilized. By the simulation experimental results, the validity of this method for magnetic flywheel storage system was demonstrated.

\section{REFERENCES}

[1] Y. Arai, H. Seino, and K. Yoshizawa, K. Nagashima, "Development of superconducting magnetic bearing with superconducting coil and bulk superconductor for flywheel energy storage system," Physica C: Superconductivity, vol. 494, no. 15, pp. 250-254, 2013.

[2] Trong Duy Nguyen, and King Jet Tseng, Chi Zhang, Shao Zhang, Hoan Thong Nguyen. "Position Sensorless Control of a Novel Flywheel Energy Storage System," IPEC, 2010 Conference Proceedings, pp. 1192-1198.

[3] Hongryel Kim, and Jubum Son, Jangmyung Lee. "A High-Speed Sliding-Mode Observer for the Sensorless Speed Control of a PMSM," IEEE Transactions on Industrial Electronics, vol. 58, no. 9, pp. 40694077, Sep 2011.

[4] SiYi Chen, Ying Luo, YouGuo Pi, "PMSM sensorless control with separate control strategies and smooth switch from low speed to high speed," ISA Transactions, vol. 58, pp. 650-658, 2015.

[5] Xiaoguang Zhang, and Zhengxi Li. "Sliding-Mode Observer-Based Mechanical Parameter Estimation for Permanent Magnet Synchronous Motor," IEEE Transactions on Power Electronics, vol. 31, no. 8, pp. 5732-5745, Aug 2016.

[6] Ren Yuan, Dan Su, and Jiancheng Fang, "Whirling modes stability criterion for a magnetically suspended flywheel rotor with signiticant gyroscopic effects and bending modes," IEEE Transactions on Power Electronics, vol. 28, no. 12, pp. 5890-5901, Dec 2013.

[7] Yoon-Seok Han, Jung-Soo Choi, and Young-Seok Kim, "Sensorless PMSM drive with a sliding mode control based adaptive and stator resistance estimator," IEEE Transactions on Magnetics, vol. 36, no. 5, pp 3588-3591, Sep 2000.

[8] [8] Jun-Jie Ren, Yan-Cheng Liu, Ning Wang, "Sensorless control of ship propulsion interior permanent magnet synchronous motor based on a new sliding mode observer," ISA Transactions, vol. 54, pp. 15-26, 2015.

[9] Hyun Lee, Jangmyung Lee, "Design of iterative sliding mode observer for sensorless PMSM control," IEEE Transactions on Control Systems Technology, vol. 21, no. 4, July 2013.

[10] Marwa Ezzat, Alain Glumineau, and Frank Plestan, "Sensorless speed control of a permanent magnet synchronous motor: high order sliding mode controller and sliding mode observer," IFAC Proceedings Volumes, vol. 43, no. 14, pp. 1290-1295, Sep 2010. 
[11] Ran Li, Guangzhou Zhao, "Position Sensorless Control for PMSM Using Sliding Mode Observer and Phase-Locked Loop," Power Electronics and Motion Control Conference, pp.1867-1870, May 2009.

[12] Chu Jianbo, Hu Yuwen, Huang Wenxin, Wang Mingjin, Yang Jianfei, and Shi Yuxia, "An improved sliding mode observer for position sensorless vector control drive of PMSM," Power Electronics and Motion Control Conference, pp. 1898-1902, May 2009.

[13] R.Krishnan, "Permanent Magnet Synchronous and Brushless DC Motor Drives," CRC Press, pp. 227-230, 2010.

[14] Xinda Song, Jiancheng Fang, Bangcheng Han, and Shiqiang Zheng, "Adaptive Compensation Method for High-Speed Surface PMSM Sensorless Drives of EMF-Based Position Estimation Error," IEEE Transactions on power electronics, vol. 31, no. 2, pp. 1438-1449, 2016.

[15] Qiao ZW, Shi TN, Wang YD, "New sliding-mode observer for position sensorless control of permanent-magnet synchronous motor," IEEE Trans Ind Electron, vol. 60, no. 2, pp. 710-719, 2013. 